\title{
Surgical techniques: robotic bladder diverticulectomy with the da Vinci-S surgical system
}

\author{
Ranjit Rao $\cdot$ Rishi Nayyar $\cdot$ S. Panda $\cdot$ Ashok K. Hemal
}

Received: 17 May 2007 / Accepted: 4 June 2007 / Published online: 30 June 2007

(C) Springer London 2007

\begin{abstract}
Bladder diverticulectomy is a surgical operation for symptomatic or large bladder diverticula. Typically, bladder diverticula are because of infravesical obstruction, although congenital diverticula can occur that may be large and symptomatic. The ability to excise the diverticulum completely, avoid important adjacent structures, and close the bladder defect in a watertight fashion are key fundamentals to this operation. Traditionally done via an open extravesical, intravesical, or combined approach, bladder diverticulectomy can now be done in a minimally invasive fashion. Both laparoscopic and robotassisted methods have clear advantages over open surgery, including smaller incision, reduced pain, improved cosmesis, and reduced blood loss, with an equivalent functional result. Large bladder diverticula, particularly those involving the ureteric orifice which required ureteric reimplantation, were often considered beyond the scope of conventional laparoscopy. Recently, use of robotic technology as a means of facilitating laparoscopic excision of bladder diverticula has provided the ability to treat large and more complex diverticula. Advantages of the robotic approach are the finer precision and dexterity of the instruments coupled with three-dimensional imaging. Although there are several case reports describing pure laparoscopic diverticulectomy, as far as we are aware there are no published reports of robotic bladder diverticulectomy. This paper will outline a safe and reproducible surgical tech-
\end{abstract}

R. Rao $\cdot$ R. Nayyar $\cdot$ S. Panda $\cdot$ A. K. Hemal $(\bowtie)$

Department of Urology,

All India Institute of Medical Sciences,

New Delhi 110029, India

e-mail: ranjit.rao@hotmail.com nique for performing robotic bladder diverticulectomy using the da Vinci-S surgical system.

Keywords Laparoscopy $\cdot$ Robot $\cdot$ Bladder diverticulum · Bladder diverticulectomy

\section{Introduction}

Bladder diverticula can be classified as congenital or acquired with the latter being more common in the older population, with infravesical obstruction related to benign prostatic hyperplasia or urethral stricture disease [1]. In the younger group, posterior urethral valves and neurogenic bladder should be excluded. Congenital bladder diverticula are uncommon but can be large and rarely present as urinary retention $[2,3]$.

Although laparoscopic surgery is feasible for bladder diverticulectomy, it remains a technically demanding procedure. The ability to excise the diverticulum and repair the bladder defect in a watertight fashion while avoiding other adjacent structures is essential to obtaining a good result. Consequently most surgeons would tend to offer patients an open operation rather than a minimally invasive one.

Use of robot-assisted technology may provide a means of overcoming the challenges faced with pure laparoscopy. The greater ease of movement and precision of suturing combined with $3 \mathrm{D}$ vision enables the surgeon to replicate open surgical techniques more accurately.

\section{Materials and methods}

We performed three robotic bladder diverticulectomies during the period January-May 2007. Before surgery, work up 
included urinalysis, full blood count, electrolytes, and creatinine. Imaging included renal tract ultrasound, intravenous pyelogram, and CT cystogram with $3 \mathrm{D}$ reconstructions.

Cystoscopy was performed to exclude anatomical obstruction and to give the exact size and location of the diverticulum and the proximity to the ureter (Fig. 1).

\section{Results}

Patients were aged 16, 20, and 58. The mean operating time was $90 \mathrm{~min}$ and the average blood loss was $50 \mathrm{~mL}$. The mean length of stay was 3 days. All patients had their catheters removed on day 10 after a cystogram confirmed there was no leak (Fig. 2).

As in any surgical procedure the preoperative work-up should be complete. Before bladder diverticulectomy the cystogram and cystoscopic findings should be reviewed to

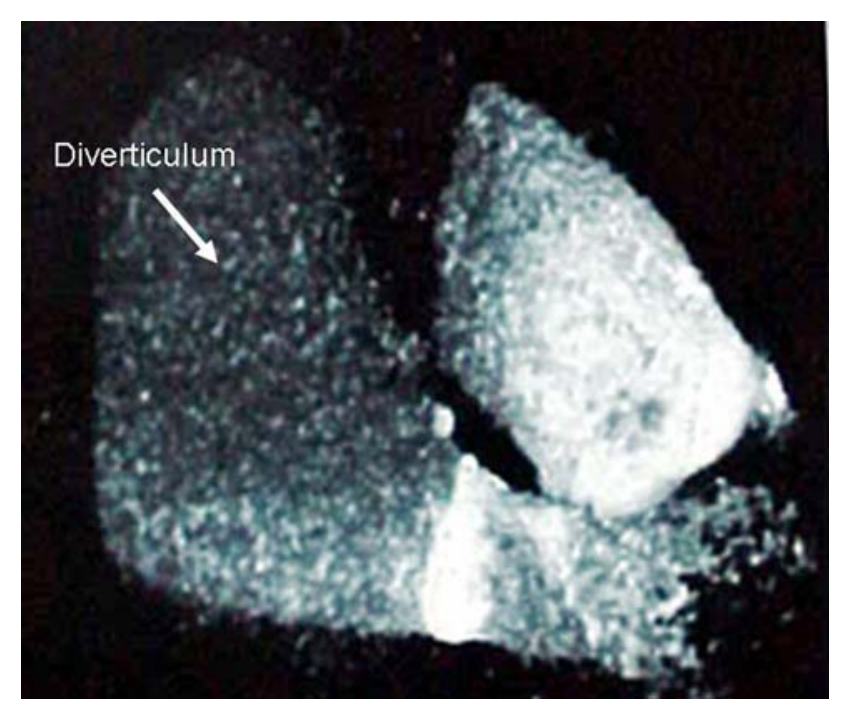

Fig. 1 CT cystogram

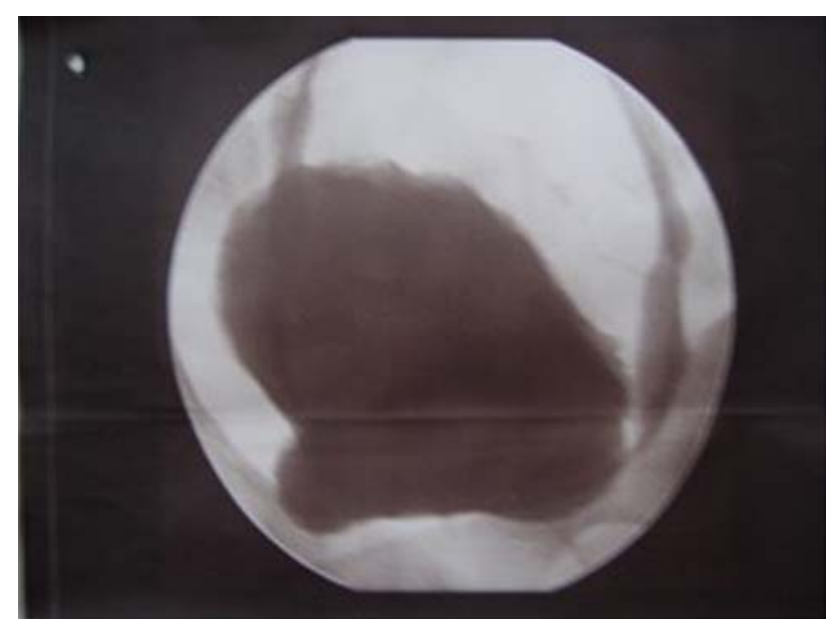

Fig. 2 Post op cystogram confirm size, position, and relationships to ureters, vas deferens, and rectum because these structures are at risk, particularly with posteriorly located diverticula. The surgeon should be prepared and able to perform ureteric reimplantation if the diverticulum involves the ureter.

\section{Robotic bladder diverticulectomy technique}

Step 1: patient position

For robotic diverticulectomy patients are placed in a low dorsal lithotomy position. The arms are padded with cotton and tucked by the patients' side and all pressure points are carefully cushioned. This reduces the risk of neuropraxia and other pressure-related problems. To prevent the patient from slipping when in the Trendelenburg position we use an anti-slip rubber mat and strapping.

Step 2: placement of ureteric catheter or JJ stent

As in open surgery, a ureteric catheter or JJ stent is placed particularly if the diverticulum is in close proximity to the ureteric orifice.

\section{Step 3: port position and pneumoperitoneum}

Unlike the standard method of creating a pneumoperitoneum with either Veres needle or open Hassan technique, we have been using a direct insertion technique with a $12 \mathrm{~mm}$ Ethicon port. An infraumbilical incision is made through the skin and superficial fascia. The skin is held up vertically by means of either the assistants' hands or two towel clips on either side of the umbilicus. The port is directed perpendicular to the skin in a gentle rotating motion. Usually two discrete moments of "give" are felt as the port enters the peritoneal cavity. Although we have found this to be extremely safe and quick, we do not advise direct insertion if the patient has had previous abdominal surgery.

Two 8-mm robotic ports are placed in the mid-clavicular line inferolateral to the camera port just lateral to the rectus muscle. Two 5-mm ports are placed for the single assistant for suction and retraction. The robot is then docked. With good team-work this whole process of port insertion, pneumoperitoneum, and docking takes less than $10 \mathrm{~min}$ (Fig. 3).

Step 4: identification of diverticulum

The bladder is accessed via a transperitoneal route. We fill the bladder via a 16 Fr IDC and the bulge of the diverticulum is visualized easily. The peritoneum over the bladder is then taken down to expose the diverticulum. The bladder is 


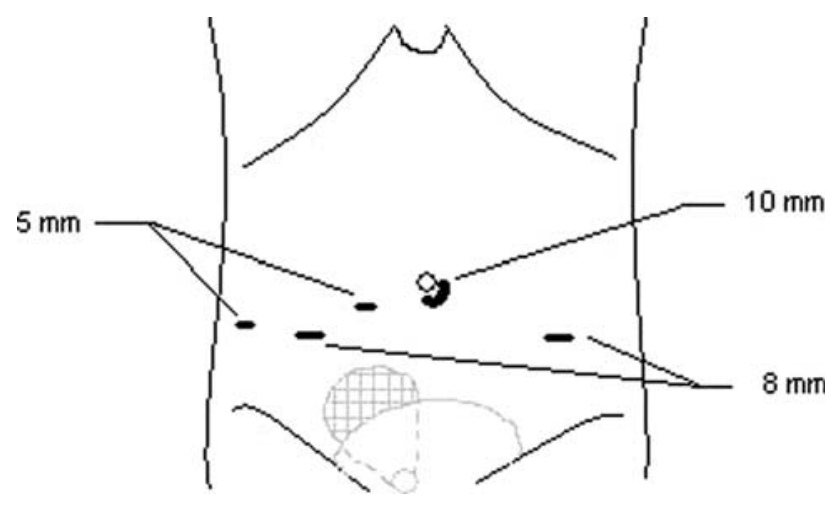

Fig. 3 Port positions

then be emptied via the catheter and using a combination of sharp and blunt dissection the diverticulum is dissected to its neck.

\section{Step 5: robotic diverticulectomy}

Meticulous dissection is crucial to avoid injury to the ureters, vas deferens, and rectum. Once the diverticulum has been dissected all the way to its neck it is then excised (Fig. 4).

\section{Step 6: closure of bladder defect}

The dome of the bladder is hitched anteriorly with a retraction suture to the anterior abdominal wall to aid visualization of the bladder defect. The bladder is closed in two layers with 30 Vicryl cut to 6 in. The EndoWrist instruments make dissection and intracorporeal suturing a

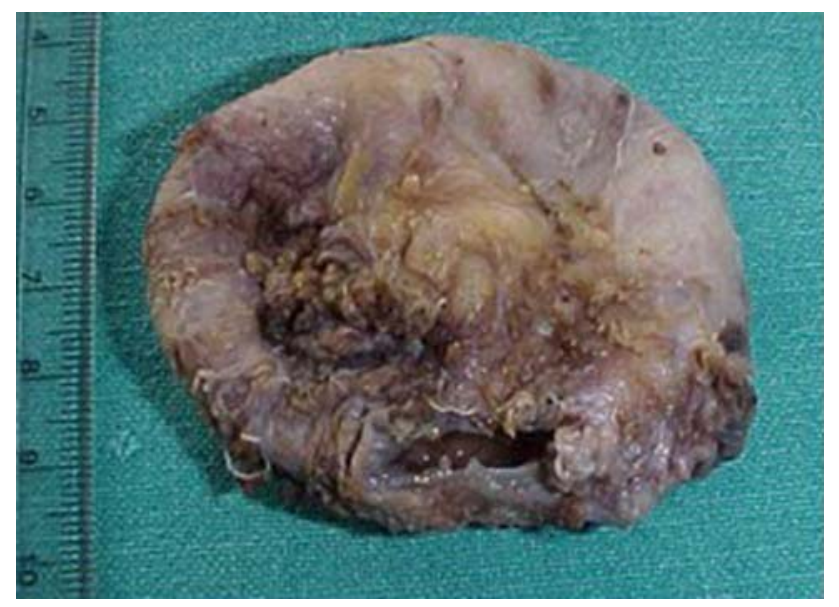

Fig. 4 Diverticulum specimen straightforward procedure and the pneumperitoneum reduces bleeding.

Step 7: reconstitution of the retropubic space

The peritoneum over the bladder is reconstituted to recreate the retropubic space.

Step 8: specimen retrieval

A $5 \mathrm{~mm}$ laparoscopic telescope is introduced via a $5 \mathrm{~mm}$ port. A grasper placed through the camera port grasps the diverticulum. The diverticulum specimen is retrieved and sent for histopathology. A $16 \mathrm{Fr}$ in-dwelling catheter is left in-situ.

Step 9: port closure

All instruments and ports are removed and the port sites closed appropriately.

A cystogram is performed on day 10 to exclude any leak, after which the catheter is removed.

\section{Discussion}

Congenital bladder diverticula are uncommon [2, 3]. In rare circumstances they can cause urinary obstruction [4]. These diverticula are not caused by outflow obstruction but are because of a congenital weakness of the muscle layers. There are case reports associated with Ehlers Danlos syndrome in which there is an abnormality of the connective tissue [5].

As these diverticula enlarge the ureteral hiatus may become incorporated within it, a factor that must be considered when planning surgery. Although most of these diverticula remain asymptomatic, they can cause vesicoureteric reflux, urinary tract infections, stone formation and even incontinence. Malignancy can also arise within diverticula.

It is usually recommended that large or symptomatic diverticula be treated by surgical excision. Traditionally this was done by an open extravesical, intravesical, or combined approach; there are, however, many reports showing that diverticulectomy can be performed safely either laparoscopically or with robotic assistance [6-8].

We have found that the robotic approach to bladder diverticulectomy to be highly satisfactory. The patients experience the full benefits of a minimally invasive procedure - smaller size of incision, reduced pain and blood loss, and shorter hospital stay. Use of the robot makes the surgery ergonomically easier for the surgeon while providing 
greater ease of dissection and suturing compared with pure laparoscopy.

\section{Conclusion}

We have confirmed that robotic diverticulectomy can be performed safely providing the maximum benefits seen with open surgery but with the minimal morbidity of laparoscopic surgery.

\section{References}

1. Wein et al (2002) Campbell's urology, 9th edn, chap 121, pp 35793580
2. Verghese M, Belman AB (1984) Urinary retention secondary to congenital bladder diverticula in infants. J Urol 132:1186

3. Sheldon CA, Essig KA (1994) Congenital bladder diverticulum causing bladder outlet obstruction: case report and review of the literature. Pediatr Surg Int 9:141

4. Zia-ul-Miraj M (1999) Congenital bladder diverticulum: a rare cause of bladder outlet obstruction in children. J Urol 162:2112

5. Burrows NP, Monk BE, Harrison JB, Pope FM (1998) Giant bladder diverticulum in Ehlers-Danlos syndrome type I causing outflow obstruction. Clin Exp Dermatol 23:109

6. Faramarzi-Roques R, Calvet C, Gateau T, Ballanger PH (2004) Surgical treatment of bladder diverticula: laparoscopic approach. J Endourol 18(1):69-72

7. Parra RO (1992) Laparoscopic diverticulectomy: preliminary report of a new approach for the treatment of bladder diverticulum. J Urol 148(3):869-871

8. Edward G, Myer MD, Joseph R, Wagner MD (2006) Robotic-assisted laparoscopic bladder diverticulectomy. Poster presentation at the New England Section of the AUA. Hartford Hospital/University of Connecticut, Hartford 